\title{
PRINCIPAL LOCAL IDEALS IN WEIGHTED SPACES OF ENTIRE FUNCTIONS
}

\author{
BY \\ JAMES J. METZGER( $\left.{ }^{1}\right)$
}

\begin{abstract}
This paper deals with principal local ideals in a class of weighted spaces of entire functions of one variable. Let $\rho>1$ and $q>1$, and define $E_{I}[\rho, q]$ (respectively, $\left.E_{P}[\rho, q]\right)$ to be the space of all entire functions $f$ of one variable which satisfy $|f(x+i y)|$ $=O\left(\exp \left(A|x|^{\rho}+A|y|^{q}\right)\right)$ for some (respectively, all) $A>0$. It is shown that in each of the spaces $E_{I}[\rho, q]$ and $E_{\mathrm{P}}[\rho, q]$, the local ideal generated by any one function coincides with the closed ideal generated by the function. This result yields consequences for convolution on these spaces. It is also proved that when $\rho \neq q$ a division theorem fails to hold for either space $E_{I}[\rho, q]$ or $E_{P}[\rho, q]$.
\end{abstract}

1. Introduction. In this paper we study aspects of the ideal structure of certain locally convex rings of entire functions of one variable. We begin by introducing these rings and indicating the type of problem to be studied.

Definition 1.1. Let $\varphi: \boldsymbol{C} \rightarrow \boldsymbol{R}$ be continuous. For $f: \boldsymbol{C} \rightarrow \boldsymbol{C}$, write

$$
\|f\|_{\varphi}=\sup \{|f(z)| \exp (-\varphi(z)): z \in C\} \text {. }
$$

Define $E(\varphi)$ to be the collection of all entire functions $f$ such that $\|f\|_{\varphi}<\infty$.

Note that under \|\|$_{\varphi}, E(\varphi)$ is a Banach space whose topology is finer than the topology of uniform convergence on compact sets.

Definition 1.2. Let $\rho>0$ and $q>0$. Define

$$
E_{I}[\rho, q]=\bigcup_{A>0} E\left(A|x|^{\rho}+A|y|^{q}\right) \quad \text { and } \quad E_{P}[\rho, q]=\bigcap_{A>0} E\left(A|x|^{\rho}+A|y|^{q}\right),
$$

where $z=x+i y$.

The spaces $E_{I}=E_{I}[\rho, q]$ and $E_{P}=E_{P}[\rho, q]$ are locally convex topological vector spaces over $C$, where $E_{I}$ is given the inductive limit topology from the Banach spaces $E\left(A|x|^{\rho}+A|y|^{q}\right)$, and $E_{P}$ is given the corresponding projective limit topology (Schaefer [10]). The topology on each of $E_{I}$ and $E_{P}$ is finer than the topology of uniform convergence on compact sets. Also, each of $E_{I}$ and $E_{P}$ is a ring whose multiplication is continuous; that is, for fixed $f$ in the space, the mapping $g \rightarrow g f$ (multiplication) on the space is continuous.

Received by the editors May 15, 1971 and, in revised form, July 21, 1971.

AMS 1970 subject classifications. Primary 46E10, 46E25, 46H10, 30A64; Secondary $31 \mathrm{C} 05,44 \mathrm{~A} 35$.

Key words and phrases. Local ideals, locally convex spaces, weighted spaces of entire functions, convolution.

(1) This article is part of the author's doctoral dissertation at the University of Michigan under the supervision of Professor B. A. Taylor. The author wishes to thank Professor Taylor for his help and encouragement.

Copyright (C) 1972, American Mathematical Society 
Definition 1.3. Let $\rho>0$ and $q>0$, let $E$ denote either space $E_{I}[\rho, q]$ or $E_{P}[\rho, q]$, and let $f \in E, f \not \equiv 0$. Define $I_{\mathrm{loc}}(f)=\{g \in E: g / f$ is entire $\}$. Also, write $I(f)^{-}=\{h f: h \in E\}^{-}$, the closure in $E$ of the ideal generated by $f$.

The set $I_{\text {loc }}(f)$ is a closed ideal in $E$ containing $f$, and is referred to as the local ideal in $E$ generated by $f$; whereas, $I(f)^{-}$is the closed ideal in $E$ generated by $f$.

The problem we wish to consider is the following: in the spaces $E=E_{I}[\rho, q]$ and $E=E_{P}[\rho, q]$, do the two closed ideals $I_{\mathrm{loc}}(f)$ and $I(f)^{-}$generated by a function $f \in E$ necessarily coincide.

This problem is a special case of the more general problem where rings of analytic functions of $n$ variables are considered and the local (respectively, closed) ideals are generated by sets of functions rather than just one function. The general problem has been studied extensively by such authors as L. Ehrenpreis [4], [5], B. Malgrange [7], L. Schwartz [11], H. Cartan [2], and D. J. Newman and H. S. Shapiro [8].

Let $\rho$ and $q$ be positive numbers, at least one of which is greater than 1 . In $\S 2$ it is shown that, in each of the spaces $E=E_{I}[\rho, q]$ and $E=E_{P}[\rho, q], I_{\mathrm{loc}}(f)=I(f)^{-}$for all $f \in E, f \not \equiv$. One technique for proving this would be to show that a "division theorem" holds for the space $E$; that is, if $f \in E$ and $g \in E$, with $f \not \equiv 0$ and $g / f$ entire, then $g / f \in E$. It is well known that such a division theorem does indeed hold for both $E=E_{I}[\rho, q]$ and $E=E_{P}[\rho, q]$ in the case $\rho=q$. However, when $\rho \neq q$ such a division theorem fails to hold for either $E=E_{I}[\rho, q]$ or $E=E_{P}[\rho, q]$, as will be proved in $\$ 3$.

Lastly, when both $\rho$ and $q$ are greater than 1 , the identification of ideals $I_{100}(f)$ and $I(f)^{-}$in $E_{I}[\rho, q]$ and $E_{P}[\rho, q]$ has consequences for convolution in the spaces. These consequences are noted in $\$ 4$.

2. Main results. In this section the results about local ideals in $E_{I}$ and $E_{P}$ are proved.

THEOREM 2.1. Let $\rho>0$ and $q>0$, with $\rho \geqq 1$ or $q \geqq 1$. Then $I_{\mathrm{loc}}(f)=I(f)^{-}$in $E_{I}[\rho, q]$ for each $f \in E_{I}[\rho, q], f \not \equiv$.

THEOREM 2.2. Let $\rho>0$ and $q>0$, with $\rho>1$ or $q>1$. Then $I_{\mathrm{loc}}(f)=I(f)^{-}$in $E_{P}[\rho, q]$ for each $f \in E_{P}[\rho, q], f \not 0$.

Both theorems will be obtained as consequences of Theorem 2.3 below. For the statement of this result, additional notation is required.

Definition 2.1. For $\varphi: \boldsymbol{C} \rightarrow \boldsymbol{R}$ continuous, define $\mathfrak{A}(\varphi)$ to be the collection of all entire functions $f$ that are square-integrable with respect to the measure $\exp (-\varphi) d \lambda$, i.e.

$$
\int_{C}|f(z)|^{2} \exp (-\varphi(z)) d \lambda(z)<\infty .
$$

Here $\lambda$ denotes Lebesgue measure on $\boldsymbol{C}=\boldsymbol{R}^{2}$. The space $\mathfrak{A}(\varphi)$ is a Hilbert space with the usual inner product. 
Also, we write $\log ^{+} a$ for $\max \{\log a, 0\}$ if $a \geqq 0$.

THEOREM 2.3. Let $\rho \geqq 1$ and let $f$ and $g$ be in $E\left(A|z|^{\rho}\right)$, where $A>0$, and assume $g / f$ is entire. Then there exists a sequence $\left\{G_{j}\right\}$ of entire functions such that $G_{j} f$ (multiplication) converges to $g$ in the space $\mathfrak{A}(\varphi)$, where

$$
\varphi(z) \equiv M A|x|^{\rho}+3 \log \left(1+|z|^{2}\right)+2 \max \left\{\log ^{+}|f(z)|, \log ^{+}|g(z)|\right\},
$$

and where $M>0$ depends only on $\rho$. Further, the functions $G_{j}$ may be chosen such that

$$
G_{j} \in \mathfrak{A}\left(A_{j}|x|+\log \left(1+|z|^{2}\right)\right), \quad j=1,2, \ldots
$$

The proof of Theorem 2.3 is based on a result of B. A. Taylor [13]. We state that result here for the one variable case:

Proposition 2.1. Let $\chi, \chi_{1}, \chi_{2}, \ldots$, be continuous (real-valued) subharmonic functions on $C$ with $\chi_{m}$ increasing pointwise to $\chi$ as $m \rightarrow \infty$. Then the closure of $\bigcup_{m} \mathfrak{A}\left(\chi_{m}+\log \left(1+|z|^{2}\right)\right)$ in the Hilbert space $\mathfrak{A}\left(\chi+\log \left(1+|z|^{2}\right)\right)$ contains $\mathfrak{A}(\chi)$.

The method of proof of Theorem 2.3 is to construct subharmonic functions $\chi, \chi_{1}, \chi_{2}, \ldots$, which satisfy the hypotheses of Proposition 2.1 , and to choose a $\varphi$ of the form (2.1), such that the following inequalities hold:

$$
\begin{aligned}
& \text { (a) } 2 \log |(g \mid f)(z)|+2 \log \left(1+|z|^{2}\right) \leqq \chi(z)+K, \\
& \text { (b) } \chi_{m}(z) \leqq K_{m}|x|+K_{m}^{\prime}, \\
& \text { (c) } \chi(z)+\log \left(1+|z|^{2}\right)+2 \log |f(z)| \leqq \varphi(z)+K^{\prime},
\end{aligned}
$$

for all $z \in C$, where $K, K^{\prime}, K_{m}, K_{m}^{\prime}$ denote constants. Then $g / f$ will be in $\mathfrak{A}(\chi)$ by (a), and Proposition 2.1 will imply the existence of a sequence $\left\{G_{j}\right\}$ in

$$
\bigcup_{m} \mathfrak{A}\left(\chi_{m}+\log \left(1+|z|^{2}\right)\right)
$$

which converges to $g / f$ in $\mathfrak{A}\left(\chi+\log \left(1+|z|^{2}\right)\right)$. The functions $G_{j}$ will satisfy (2.2) by (b), and $G_{j} f$ will converge to $g$ in $\mathfrak{A}(\varphi)$ by (c). This will suffice for the theorem.

REMARK. The inequalities (2.3)(a) and (2.3)(c) could be satisfied by taking

$$
\begin{aligned}
& \chi(z) \equiv 2 \log ^{+}|(g / f)(z)|+2 \log \left(1+|z|^{2}\right), \\
& \varphi(z) \equiv 3 \log \left(1+|z|^{2}\right)+2 \max \left\{\log ^{+}|f(z)|, \log ^{+}|g(z)|\right\} .
\end{aligned}
$$

However, in order to choose subharmonic functions $\chi_{m}$ which converge upward to $\chi$ and satisfy (2.3)(b), we have found it necessary to take somewhat larger functions $\chi$ and $\varphi$.

We now prove a lemma which will be used to construct appropriate subharmonic functions $\chi_{m}$.

LEMMA 2.1. Let $\rho>0$ and $G \in E\left(A|z|^{\rho}\right)$ for some $A>0$. Also let

$$
0<\alpha<\min \{\pi / 2 \rho, \pi\} \text {. }
$$

Then there exists a sequence $\left\{u_{m}\right\}$ of functions subharmonic in the sector

$$
S=\left\{r e^{i \theta}: 0<r<\infty,(\pi / 2)-\alpha<\theta<(\pi / 2)+\alpha\right\},
$$

such that each $u_{m}$ is bounded above in $S$, and such that the functions $u_{m}$ increase pointwise in $S$ to $\log |G|$ as $m \rightarrow \infty$. 
Proof. By the analytic change of coordinates $w=i(-i z)^{\delta}$ (using the principal branch of the logarithm) with $\delta=2 \alpha / \pi$, the function $G$ on the sector $S$ defines an analytic function $g(z)=G(w)$ in the upper half-plane $\mathscr{H}$. We will exhibit functions $v_{m}, m=1,2, \ldots$, on $\mathscr{H}$ which are subharmonic, bounded above, and pointwise increasing to $\log |g|$. The desired subharmonic functions $u_{m}$ can then be obtained via the inverse change of coordinates.

We will use a representation theorem for functions of exponential type in $\mathscr{H}$ to construct suitable functions $v_{m}$. Note first that $g$ is analytic in $\mathscr{H}$ and continuous in the closed half-plane $\mathscr{H}^{-}$. Also, the zeros of $g$ have no finite limit point. Since $G \in E\left(A|z|^{\circ}\right)$, there exists $C>0$ such that

$$
\log |g(z)| \leqq A|z|^{\delta \rho}+C
$$

for all $z \in \mathscr{H}^{-}$. This implies that $g$ is of exponential type in $\mathscr{H}$, since $\delta \rho=2 \alpha \rho / \pi<1$. It follows then (Boas [1, Theorem 6.5.4]) that $g$ has the following representation in the upper half-plane:

$$
\log |g(z)|=y \pi^{-1} \int_{-\infty}^{\infty}\left[(t-x)^{2}+y^{2}\right]^{-1} \log |g(t)| d t+\log |B(z)|+\gamma y
$$

for all $z=x+i y \in \mathscr{H}$. Here $B$ is the Blaschke product associated with $g$. It is an analytic function in $\mathscr{H}$ and satisfies $|B(z)| \leqq 1$ for all $z \in \mathscr{H}$. The constant $\gamma$ is defined by

$$
\gamma=\lim _{r \rightarrow \infty}\left\{(2 / \pi) r^{-1} \int_{0}^{\pi}(\sin \theta) \log \left|g\left(r e^{i \theta}\right)\right| d \theta\right\}
$$

Note that $\gamma \leqq 0$ because of (2.4).

Now, for $m=1,2, \ldots$, define

$$
v_{m}(z)=y \pi^{-1} \int_{-\infty}^{\infty}\left[(t-x)^{2}+y^{2}\right]^{-1} h_{m}(t) d t+\log |B(z)|+\gamma y
$$

for all $z \in \mathscr{H}$, where

$$
\begin{aligned}
h_{m}(t) & =\log |g(t)| & & \text { if } \log |g(t)| \leqq m, \\
& =m & & \text { if } \log |g(t)| \geqq m .
\end{aligned}
$$

It is readily verified that each $v_{m}$ is a well-defined subharmonic function in $\mathscr{H}$ satisfying $v_{m}(z) \leqq m$ for all $z \in \mathscr{H}$, and that the functions $v_{m}$ increase pointwise to $\log |g|$ as $m \rightarrow \infty$. This completes the proof of the lemma.

Proof of Theorem 2.3. As indicated earlier, it suffices to construct subharmonic functions $\chi, \chi_{1}, \chi_{2}, \ldots$, satisfying the hypotheses of Proposition 2.1, together with a function $\varphi$ of the form (2.1), such that the inequalities (2.3) hold. Throughout the proof, we use $M_{1}, M_{2}$, etc., to denote positive constants which depend only on $\rho$.

Since $f$ and $g$ are in $E\left(A|z|^{\circ}\right)$ and since $g / f$ is entire, it follows by a well-known result (see, e.g., Taylor [12, Lemma 7.2]) that $g / f \in E\left(M_{1} A|z|^{\circ}\right)$. For $z \in C$, define

$$
G(z)=z^{4}[(g / f)(z)]^{2} .
$$


The function $G$ also has a bound given by $G \in E\left(M_{2} A|z|^{\rho}\right)$.

Consider now two subsets of $C$ :

$$
S=\left\{r e^{i \theta}: 0<r<\infty,(\pi / 2)-(\pi / 3 \rho)<|\theta|<(\pi / 2)+(\pi / 3 \rho)\right\}
$$

and

$$
\begin{aligned}
S_{1}=\left\{r e^{i \theta}: 0 \leqq r<1\right. & \text { or }(-\pi / 2)+(\pi / 4 \rho)<\theta<(\pi / 2)-(\pi / 4 \rho) \\
& \text { or }(\pi / 2)+(\pi / 4 \rho)<\theta<(3 \pi / 2)-(\pi / 4 \rho)\} .
\end{aligned}
$$

Applying Lemma 2.1 in turn to $G(z)$ and $G(-z)$, it follows that there exists a sequence $\left\{u_{m}\right\}$ of functions subharmonic in $S$ and bounded above in $S$ which increase pointwise in $S$ to $\log |G|$. On the second set $S_{1}$, the function $G$ satisfies

$$
\log |G(z)| \leqq M_{3} A|x|^{\rho}+C
$$

for all $z=x+i y \in S_{1}$, where $C$ is positive constant which need not depend only on $\rho$.

Now choose a sequence $\left\{v_{k}\right\}$ of convex functions on $\boldsymbol{R}$ such that, as $k \rightarrow \infty, v_{k}(x)$ increases to $M_{3} A|x|^{\rho}$ uniformly on compact subsets of $\boldsymbol{R}$, and such that

$$
\begin{array}{ll}
v_{k}(x) \leqq \alpha_{k}|x|+\beta_{k} & \text { for all } x \in R \text { and } k=1,2, \ldots, \\
v_{1}(x) \geqq \varepsilon|x|+\beta & \text { for all } x \in R,
\end{array}
$$

where $\varepsilon>0$ and $\beta \in \boldsymbol{R}, \alpha_{k}>0$ and $\beta_{k} \in \boldsymbol{R}$ for $k=1,2, \ldots$ For example,

$$
\begin{aligned}
v_{k}(x) & =M_{3} A|x|^{\rho} & & \text { if }|x| \leqq k, \\
& =M_{3} A k^{\rho-1}(\rho|x|+k-\rho k) & & \text { if }|x| \geqq k .
\end{aligned}
$$

The sequences $\left\{u_{m}\right\}$ and $\left\{v_{k}\right\}$ will be used to construct the desired sequence of subharmonic functions $\chi_{m}$.

Let $m$ be a fixed positive integer. The function $u_{m}$ is bounded above on $S$ and hence on $S \cap S_{1}$. Consequently, $u_{m}(z) \leqq v_{1}(x)$ for $z=x+i y \in S \cap S_{1}$ with $|x|$ sufficiently large. But on $S \cap S_{1}, u_{m}(z) \leqq \log |G(z)| \leqq M_{3} A|x|^{\rho}+C$. Since $v_{k}(x)$ $\rightarrow M_{3} A|x|^{\rho}$ as $k \rightarrow \infty$, uniformly on compact sets, it follows that there exists $k=k(m)$ such that

$$
u_{m}(z) \leqq v_{k}(x)+C+1
$$

for those $z \in S \cap S_{1}$ for which $u_{m}(z)>v_{1}(x)$. Since $v_{k}(x) \geqq v_{1}(x)$ for all $x$ and since $C>0$, we conclude that (2.6) holds everywhere in $S \cap S_{1}$. Also, we may assume that $k=k(m)$ is chosen so that $k(m)>k(m-1), m=2,3, \ldots$

Now, for $m=1,2, \ldots$, define

$$
\begin{aligned}
\chi_{m}(z) & =v_{k}(x)+C+1 & & \text { if } z \in S_{1}, \\
& =\max \left\{v_{k}(x)+C+1, u_{m}(z)\right\} & & \text { if } z \in S,
\end{aligned}
$$

where $k=k(m)$. Note from (2.6) that the two definitions agree on $S \cap S_{1}$. The function $\chi_{m}$ is defined everywhere on $C$ and is subharmonic. Also, since $\left\{u_{m}\right\}$ increases pointwise to $\log |G|$ and $\left\{v_{k}\right\}$ increases pointwise to $M_{3} A|x|^{\circ}$, and since $G$ satisfies (2.5), it follows that $\left\{x_{m}\right\}$ increases pointwise to the subharmonic function

$$
\chi(z) \equiv \max \left\{M_{3} A|x|^{\rho}+C+1, \log |G(z)|\right\} .
$$


The functions $\chi, \chi_{1}, \chi_{2}, \ldots$, thus satisfy the hypotheses of Proposition 2.1.

In order to complete the proof, we note the following: The definitions of $\chi$ and $G$ imply that (2.3)(a) holds. Condition (2.3)(b) is satisfied because of the definition of $\chi_{m}$ and the choices of $u_{m}$ and $v_{k}$. And (2.3)(c) is valid with

$$
\varphi(z) \equiv M_{3} A|x|^{\rho}+3 \log \left(1+|z|^{2}\right)+2 \max \left\{\log ^{+}|f(z)|, \log ^{+}|g(z)|\right\},
$$

which has the required form (2.1).

We now prove Theorems 2.1 and 2.2 using Theorem 2.3.

Proof of Theorem 2.1. Write $E_{I}=E_{I}[\rho, q]$ and assume that $\rho \geqq q>0$ and $\rho \geqq 1$. Since $I(f)^{-} \subseteq I_{\text {loc }}(f)$, it suffices to show that if $g \in E_{I}$ with $g / f$ entire, then $g \in I(f)^{-}$. To accomplish this we will apply Theorem 2.3 to obtain a sequence $\left\{G_{j}\right\}$ in $E_{I}$ such that $G_{j} f \rightarrow g$ in $E_{I}$ as $j \rightarrow \infty$.

Since $f, g \in E_{I}$, there exists $A>0$ such that $f, g \in E\left(A|x|^{\rho}+A|y|^{q}\right)$. Theorem 2.3 yields the existence of a sequence $\left\{G_{j}\right\}$ of entire functions satisfying (2.2) such that $G_{j} f \rightarrow g$ in $\mathfrak{A}(\varphi)$, where

$$
\varphi(z) \equiv 2 M A|x|^{\rho}+3 \log \left(1+|z|^{2}\right)+2 \max \left\{\log ^{+}|f(z)|, \log ^{+}|g(z)|\right\},
$$

and $M>0$ depends only on $\rho$. It follows that $G_{j} f \rightarrow g$ in $\mathfrak{A}\left((2 M+3) A\left(|x|^{\rho}+|y|^{q}\right)\right)$.

For $h$ entire and $w \in C$,

$$
|h(w)|^{2} \leqq \frac{1}{\pi} \int_{|z-w| \leqq 1}|h(z)|^{2} d \lambda(z)
$$

Setting $h=G_{j}$ and introducing the factor

$$
\exp \left[-\left(A_{j}|x|+\log \left(1+|z|^{2}\right)\right)\right] \exp \left[A_{j}|x|+\log \left(1+|z|^{2}\right)\right]
$$

into the integrand on the right and then applying (2.2), we conclude that $G_{j} \in E\left(\frac{1}{2} A_{j}|x|+\frac{1}{2} \log \left(1+|z|^{2}\right)\right), j=1,2, \ldots$ Thus $G_{j} \in E_{I}, j=1,2, \ldots$

A similar argument using (2.7) and the fact that $G_{j} f \rightarrow g$ in

$$
\mathfrak{A}\left((2 M+3) A\left(|x|^{\rho}+|y|^{q}\right)\right)
$$

yields that $G_{j} f \rightarrow g$ in $E\left((2 M+3) A\left(|x|^{\rho}+|y|^{q}\right)\right)$. Consequently $G_{j} f \rightarrow g$ in $E_{I}$, and the proof is complete.

Proof of Theorem 2.2. Assume $\rho \geqq q>0$ and $\rho>1$. Let $g \in E_{P}=E_{P}[\rho, q]$ with $g / f$ entire. The sets

$$
\left\{h \in E_{P}:\|h\|_{\varepsilon|x|^{\rho}+\varepsilon|y|^{q}} \leqq \delta\right\}, \quad \varepsilon>0, \delta>0,
$$

constitute a fundamental system of neighborhoods of zero in $E_{P}$. Consequently, in order to show that $g \in I(f)^{-}$it suffices to prove that for each $\varepsilon>0$ there exists a sequence $\left\{G_{j}\right\}$ in $E_{P}$ such that $G_{j} f \rightarrow g$ in $E\left(\varepsilon|x|^{\rho}+\varepsilon|y|^{q}\right)$.

For any $A>0$, the functions $f$ and $g$ are in $E\left(A|x|^{\rho}+A|y|^{q}\right)$. Then, as in the proof of Theorem 2.1, there exists a sequence of entire functions $\left\{G_{j}\right\}$ satisfying $G_{j} \in E\left(\frac{1}{2} A_{j}|x|+\frac{1}{2} \log \left(1+|z|^{2}\right)\right), j=1,2, \ldots$, such that $G_{j} f \rightarrow g$ in

$$
E\left((2 M+3) A\left(|x|^{o}+|y|^{q}\right)\right),
$$


where $M>0$ depends only on $\rho$. For $\varepsilon>0$, choose $A=\varepsilon /(2 M+3)$. Then the sequence $\left\{G_{j}\right\}$ corresponding to $A$ satisfies $G_{j} f \rightarrow g$ in $E\left(\varepsilon|x|^{\rho}+\varepsilon|y|^{q}\right)$. The bounds on the functions $G_{j}$ imply that they are in $E_{P}$, and the proof is complete.

3. Division. The main results of this section are given in the following two theorems:

THEOREM 3.1. Let $\rho>0, q>0, \rho \neq q$, with $\rho \geqq 1$ or $q \geqq 1$. Then there exist f and $g$ in $E_{I}[\rho, q]$ such that $g / f$ is entire but $g / f \notin E_{I}[\rho, q]$.

THEOREM 3.2. Let $\rho>0, q>0, \rho \neq q$, with $\rho>1$ or $q>1$. Then there exist $f$ and $g$ in $E_{P}[\rho, q]$ such that $g / f$ is entire but $g / f \notin E_{P}[\rho, q]$.

Thus a division theorem fails to hold for either $E_{I}[\rho, q]$ or $E_{P}[\rho, q]$ when $\rho \neq q$ and at least one of $\rho$ and $q$ is greater than 1. Note that the assumption $\rho \neq q$ is necessary, for a division theorem does hold for $E_{I}[\rho, q]$ and $E_{P}[\rho, q]$ when $\rho=q>0$, as is well known. Both Theorems 3.1 and 3.2 will be obtained as consequences of the following result:

THEOREM 3.3. Let $0<q<\rho$ where $\rho \geqq 1$. There exist entire functions $f$ and $g$ with $f \not \equiv$, such that $f$ and $f g$ are in $E\left(A|x|^{\rho}+A|y|^{q}\right)$ for some $A>0$, and such that

$$
\limsup _{r \rightarrow+\infty} \frac{\log |g(i r)|}{r^{\alpha}}>0
$$

where $\alpha=\rho$ if $\rho>1$ and $\alpha=(\rho+q) / 2$ if $\rho=1$.

Regarding the proof of Theorem 3.3: In the case $\rho=1$ the existence of a function $f$ of the desired form is an immediate consequence of a construction of Paley and Wiener [9] involving Fourier transforms. The function $g$ comes from an application of a result of C. O. Kiselman [6] on indicator functions.

For the case $\rho>1$, Kiselman's result will be used to prove the existence of both functions $f$ and $g$. In order to apply Kiselman's result, we give a statement of it here in a form more general than that in [6]. The proof carries over immediately.

Proposition 3.1. (See [6].) Let $\rho>0$ and let $H: C \rightarrow R$ be continuous, subharmonic, and satisfy $H($ ir $)=r^{\circ} H(i)$ for all $r>0$. Then there exists an entire function $g$ on $C$ such that $g \in E\left(\tilde{H}(z ; 3 / 2)+|z|^{q}\right)$ for all $q>0$, and such that

$$
\limsup _{r \rightarrow+\infty} \frac{\log |g(i r)|}{r^{\rho}} \geqq H(i) \text {. }
$$

Here we use the notation $\tilde{H}(z ; 3 / 2) \equiv \sup \{H(z+\zeta):|\zeta| \leqq 3 / 2\}$.

Proof of Theorem 3.3.

Case 1. $\rho=1$. Let $\alpha=(\rho+q) / 2$, and $\alpha<\beta<1$. A method of Paley and Wiener $[9$, p. 24] yields the existence of an entire function $f \not \equiv 0$ of exponential type satisfying $|f(i y)| \leqq C \exp \left(-\varepsilon|y|^{\beta}\right)$ for all $y \in R$, where $C>0, \varepsilon>0$. The function $f$ has the form $f(z) \equiv \hat{\psi}(i z)$ where ${ }^{\wedge}$ denotes Fourier transform and where $\psi$ belongs to $L_{1}(\boldsymbol{R})$, is not equivalent to zero, and is the product of two functions $\psi_{1}$ and $\psi_{2}$ with the following properties: both $\psi_{1}$ and $\psi_{2}$ are in $L_{2}(\boldsymbol{R}), \psi_{1}$ vanishes to the right of an 
$x_{0}>0$ and $\psi_{2}$ vanishes to the left of $-x_{0}$, and $\left|\hat{\psi}_{1}(x)\right|=\left|\hat{\psi}_{2}(x)\right|=\exp \left(-|x|^{\beta}\right)$ for almost all $x \in \boldsymbol{R}$.

In order to obtain the function $g$, we apply Proposition 3.1 with $\rho$ replaced by $\alpha$ and $H(z) \equiv|x|+|z|^{\alpha}$ : there exists an entire function $g \in E\left(|x|+|z|^{\alpha}+|z|^{\alpha}\right)$ which satisfies (3.1).

The functions $f$ and $f g$ are both of exponential type and bounded on the imaginary axis. Consequently, by a theorem of Phragmén and Lindelöf (see Boas [1, Theorem 1.4.3]), $f$ and $f g$ are both in $E(A|x|)$ for some $A>0$. Hence, $f$ and $f g$ are in $E\left(A|x|^{\rho}+A|y|^{q}\right)$, as sought.

Case 2. $\rho>1$. We will define functions $H_{1}$ and $H_{2}$ that satisfy the hypotheses of Proposition 3.1. First define $H_{1}$ and $H_{2}$ on the unit circle in $C$ as follows:

$$
\begin{aligned}
H_{1}\left(e^{i \theta}\right) & =A_{1} \cos \left(\rho\left(\theta-\pi / 2+\theta_{0}\right)\right) & & \text { if } \pi / 2-\theta_{0}-\pi / 2 \rho \leqq \theta \leqq \pi / 2, \\
& =A_{1} \cos \left(\rho\left(\theta-\pi / 2-\theta_{0}\right)\right) & & \text { if } \pi / 2 \leqq \theta \leqq \pi / 2+\theta_{0}+\pi / 2 \rho, \\
& =0 & & \text { if } \pi / 2+\theta_{0}+\pi / 2 \rho \leqq \theta \leqq 5 \pi / 2-\theta_{0}-\pi / 2 \rho,
\end{aligned}
$$

where $\theta_{0}=\pi / 2$ if $1<\rho \leqq 2$ and $\theta_{0}=\pi / \rho$ if $\rho \geqq 2$, and where $A_{1}>0$ is chosen so that $H_{1}\left(e^{i \theta}\right)<|\cos \theta|^{\rho}$ for all $\theta$ for which $e^{i \theta} \neq e^{-i \pi / 2}$. Also, let

$$
\begin{aligned}
H_{2}\left(e^{i \theta}\right) & =A_{2} \cos (\rho(\theta-\pi / 2)) & & \text { if } \pi / 2-\pi / 2 \rho \leqq \theta \leqq \pi / 2+\pi / 2 \rho, \\
& =0 & & \text { if } \pi / 2+\pi / 2 \rho \leqq \theta \leqq 5 \pi / 2-\pi / 2 \rho,
\end{aligned}
$$

where $A_{2}>0$ is chosen so that $H_{1}\left(e^{i \theta}\right)+H_{2}\left(e^{i \theta}\right)<|\cos \theta|^{\rho}$ for all $\theta$ for which $e^{i \theta}$ $\neq e^{-i \pi / 2}$. Both $H_{1}$ and $H_{2}$ are continuous on the unit circle. Now extend these functions to the complex plane by

$$
\begin{aligned}
H_{j}\left(r e^{i \theta}\right) & =r^{\rho} H_{j}\left(e^{i \theta}\right) & & \text { if } r>0,-\pi<\theta \leqq \pi, \\
& =0 & & \text { if } r=0,
\end{aligned}
$$

$j=1,2$. Both of the resulting functions are continuous and are positive homogeneous of order $\rho$; that is, $H_{j}(\gamma z)=\gamma^{\circ} H_{j}(z)$ for all $\gamma>0, z \in C, j=1,2$. Also, both functions are subharmonic, which follows from the fact that the functions $\theta \rightarrow H_{j}\left(e^{i \theta}\right)$ are $\rho$-subsinusoidal (see M. L. Cartwright $[3$, p. 31]).

Proposition 3.1 then implies the existence of entire functions $f$ and $g$ such that $f \in E\left(\tilde{H}_{1}(z ; 3 / 2)+|z|^{q}\right)$ and $g \in E\left(\tilde{H}_{2}(z ; 3 / 2)+|z|^{q}\right)$, with

$$
\limsup _{r \rightarrow+\infty} r^{-\rho} \log |f(i r)| \geqq H_{1}(i) \text { and } \limsup _{r \rightarrow+\infty} r^{-\rho} \log |g(i r)| \geqq H_{2}(i) \text {. }
$$

Then $f \not \equiv 0$, and $g$ satisfies (3.1) because $H_{2}(i)=A_{2}>0$.

Using the fact that $H_{1}$ and $H_{2}$ are positive homogeneous of order $\rho$, together with their definitions on the unit circle, it is readily shown that

$$
\begin{aligned}
\tilde{H}_{1}(z ; 3 / 2) & \leqq|x|^{\rho}, \\
\tilde{H}_{1}(z ; 3 / 2)+\tilde{H}_{2}(z ; 3 / 2) & \leqq|x|^{\rho},
\end{aligned}
$$

for all $z \in C$ with $|z|$ sufficiently large. From this we conclude that $f$ and $f g$ are in a space of the form $E\left(A|x|^{\rho}+A|y|^{a}\right)$, and the proof is complete. 
We now use Theorem 3.3 to prove Theorems 3.1 and 3.2.

Proof of Theorem 3.1. Without loss of generality, assume that $\rho>q$ and $\rho \geqq 1$. Theorem 3.3 implies that there exist entire functions $f$ and $g$ with $f \not \equiv 0$ and $g$ satisfying (3.1), such that $f$ and $f g$ are in $E\left(A|x|^{\rho}+A|y|^{q}\right)$ for some $A>0$. Then $f$ and $f g$ are in $E_{l}[\rho, q]$, while $f g / f=g$ cannot be in $E_{l}[\rho, q]$ because of (3.1).

Proof of Theorem 3.2. Assume $\rho>q$ and $\rho>1$. Choose $\rho^{\prime}$ and $q^{\prime}$ such that $\max \{1, q\}<\rho^{\prime}<\rho$ and $0<q^{\prime}<q$. Applying Theorem 3.3 with $\rho$ and $q$ replaced, respectively, by $\rho^{\prime}$ and $q^{\prime}$, we obtain entire functions $f$ and $g$ with $f \not \equiv 0$, such that $f$ and $f g$ are in $E\left(A|x|^{\rho^{\prime}}+A|y|^{q^{\prime}}\right)$ for some $A>0$, and

$$
\limsup _{r \rightarrow+\infty} \frac{\log |g(i r)|}{r^{\rho^{\prime}}}>0
$$

It then follows that $f$ and $f g$ are in $E_{P}[\rho, q]$ and $f g / f=g$ is not in $E_{P}[\rho, q]$.

4. Convolution. The purpose of this section is to note the consequences of Theorems 2.1 and 2.2 for convolution in the spaces $E_{I}[\rho, q]$ and $E_{P}[\rho, q]$ when $\rho>1$ and $q>1$. It is shown that in each of these spaces, every solution $f$ of a convolution equation $T * f=0$ can be approximated by finite sums of exponentialpolynomial solutions of the equation. Also, a characterization is given of those convolution operators $f \rightarrow T * f$ that map the space onto itself.

Before discussing convolution we indicate some facts about how spaces of the form $E_{I}[\rho, q]$ and $E_{P}[\rho, q]$ are related via the Laplace transformation in the case $\rho>1$ and $q>1$.

Definition 4.1. Let $\rho>1$ and $q>1$. Write $E$ for $E_{I}[\rho, q]$ or $E_{P}[\rho, q]$, and $E^{\prime}$ for its topological dual. The Laplace transform of a functional $T \in E^{\prime}$ is defined by $\check{T}(w)=T(z \rightarrow \exp (w z))$ for all $w \in C$.

Fix $\rho>1$ and $q>1$. It is readily verified that the Laplace transformation $T \rightarrow \check{T}$ maps $\left\{E_{I}[\rho, q]\right\}^{\prime}$ into $E_{P}\left[\rho^{\prime}, q^{\prime}\right]$, and $\left\{E_{P}[\rho, q]\right\}^{\prime}$ into $E_{I}\left[\rho^{\prime}, q^{\prime}\right]$, where $(1 / \rho)+\left(1 / \rho^{\prime}\right)=1$ and $(1 / q)+\left(1 / q^{\prime}\right)=1$. It will be demonstrated in a later paper that each of these mappings is a topological isomorphism of the first space onto the second, where the first is given the Mackey topology $\tau\left(E^{\prime}, E\right)$ and the second is given its natural topology. There is then a duality between $E_{I}[\rho, q]$ and $E_{P}\left[\rho^{\prime}, q^{\prime}\right]$ given by: for $f \in E_{I}[\rho, q]$ and $g \in E_{P}\left[\rho^{\prime}, q^{\prime}\right],\langle f, g\rangle=T(f)$, where $T \in\left\{E_{I}[\rho, q]\right\}^{\prime}$ with $\breve{T}=g$.

For the following definitions, let $E$ denote either $E_{I}[\rho, q]$ or $E_{P}[\rho, q]$, where $\rho>1$ and $q>1$. Then $\check{E}^{\prime}$ is, respectively, $E_{P}\left[\rho^{\prime}, q^{\prime}\right]$ or $E_{I}\left[\rho^{\prime}, q^{\prime}\right]$.

Definition 4.2. For $T \in E^{\prime}$ and $f \in E$, define the convolution $T * f$ of $f$ with $T$ by $(T * f)(\zeta)=T\left(f_{\zeta}\right)$ for all $\zeta \in C$, where $f_{\zeta}(z) \equiv f(z+\zeta)$.

It is readily shown that convolution with a functional $T \in E^{\prime}$ is a continuous operator on $E$ whose adjoint on $\breve{E}^{\prime}$ is multiplication by $\check{T}$.

Definition 4.3. An exponential-polynomial on $C$ is a function of the form $z \rightarrow P(z) e^{a z}$, where $a \in C$ and $P$ is a polynomial. 
Note that $E$ contains all exponential-polynomials.

In Theorem 4.1 below we state our results about convolution. These results follow easily from Theorems 2.1 and 2.2 and basic duality theorems for locally convex spaces.

THEOREM 4.1. Let $\rho>1$ and $q>1$, let $E$ denote either $E_{I}[\rho, q]$ or $E_{P}[\rho, q]$, and let $T \in E^{\prime}$. Then

(i) Every solution $f$ in $E$ of the convolution equation $T * f=0$ can be approximated in $E$ by finite sums of exponential-polynomial solution of the equation.

(ii) For $T \neq 0$, the convolution operator $f \rightarrow T * f$ maps $E$ onto $E$ if and only if $\check{T}$ satisfies the following condition: If $g \in \check{E}^{\prime}$ with $g / \breve{T}$ entire, then $g / \breve{T} \in \check{E}^{\prime}$.

Theorems 3.1, 3.2, and 4.1(ii) yield the following corollary:

Corollary 4.1. Let $\rho>1$ and $q>1$ with $\rho \neq q$, and let $E$ denote either $E_{I}[\rho, q]$ or $E_{P}[\rho, q]$. Then there exist $T \in E^{\prime}$ and $h \in E$ such that the convolution equation $T * f=h$ has no solution $f$ in $E$.

\section{BIBLIOGRAPHY}

1. R. P. Boas, Jr., Entire functions, Academic Press, New York, 1954. MR 16, 914.

2. H. Cartan, Idéaux et modules de fonctions analytiques de variables complexes, Bull. Soc. Math. France 78 (1950), 29-64. MR 12, 172.

3. M. L. Cartwright, Integral functions, Cambridge Tracts in Math. and Math. Phys., no. 44, Cambridge Univ. Press, Cambridge, 1956. MR 17, 1067.

4. L. Ehrenpreis, Mean periodic functions. I, Amer. J. Math. 77 (1955), 293-328. MR 16, 1122.

5. - Fourier analysis in several complex variables, Pure and Appl. Math., vol. 17, Interscience, New York, 1970.

6. C. O. Kiselman, Existence of entire functions of one variable with prescribed indicator, Ark. Mat. 7 (1969), 505-508. MR 39 \#446.

7. B. Malgrange, Existence et approximation des solutions des équations aux dérivées partielles et des équations de convolution, Ann. Inst. Fourier, Grenoble 6 (1955-1956), 271-355. MR 19, 280.

8. D. J. Newman and H. S. Shapiro, Certain Hilbert spaces of entire functions, Bull. Amer. Math. Soc. 72 (1966), 971-977. MR 34 \#4890.

9. R. E. A. C. Paley and N. Wiener, Fourier transforms in the complex domain, Amer. Math. Soc. Colloq. Publ., vol. 19, Amer. Math. Soc., Providence, R. I., 1934.

10. H. H. Schaefer, Topological vector spaces, Macmillan, New York; Collier-Macmillan, London, 1966. MR 33 \#1689.

11. L. Schwartz, Théorie générale des fonctions moyenne-périodiques, Ann. of Math. (2) 48 (1947), 857-929. MR 9, 428.

12. B. A. Taylor, Some locally convex spaces of entire functions, Entire Functions and Related Parts of Analysis (Proc. Sympos. Pure Math., La Jolla, Calif., 1966), Amer. Math. Soc., Providence, R. I., 1968, pp. 431-467.

13. - On weighted polynomial approximation of entire functions, Pacific J. Math. 36 (1971), 523-539.

Department of Mathematics, University of Georgia, Athens, Georgia 30601 